DOI: 10.12731/wsd-2018-4-88-110

УДК 616-006.6

\title{
ТАКТИКА ВЕДЕНИЯ \\ ПАЦИЕНТОВ С ДИФФУЗНЫМ РАКОМ ЖЕЛУДКА, ОБУСЛОВЛЕННЫМ НАСЛЕДСТВЕННЫМИ МУТАЦИЯМИ
}

\author{
Быков И.И., Немцова М.В., Хоробрых Т.В.
}

В структуре онкологической заболеваемости во всем мире рак желудка занимает пятое место и является третьей среди наиболее распространенных причин смерти от рака, абсолютное число смертельных случаев в год оченивается в 723 000. Подавляющее большинство случаев рака желудка имеют спорадический характер, но известно, что 1-3\% являются проявлением наследственных форм и синдромов. Настоящая статья посвящена новым тенденциям и стратегии в управлении риском развития наследственного диффузного рака желудка. Освещены основы генетического консультирования и современные критерии отбора семей, с ичелью проведения анализа наследственных мутаций. Рассмотрены вопросы эндоскопического скрининга и динамического наблюдения за состоянием слизистой оболочки желудка. Обсужден возможный подход $\kappa$ выбору тактики хирургического лечения в объеме профилактической гатсрэктомии. Обобщены особенности морфологического исследования при наследственно диффузном раке желудка. Изложены приниипь послеоперационного ухода. Рассмотрены перспективы медицинского обеспечения пачиентов с наследственным диффузным раком желудка.

Ключевые слова: наследственный рак желудка; диффузный рак желудка; мутации гена СDH1; генетическое консультирование.

\section{SURVEILLANCE OF PATIENTS WITH DIFFUSE GASTRIC CANCER ASSOCIATED WITH HEREDITARY MUTATIONS}

\section{Bykov I.I., Nemtsova M.V., Khorobrykh T.V.}

Worldwide the gastric cancer holds fifth place above all oncological diseases and the third among the most common causes of death from cancer, the total num- 
ber of deaths per year is estimated at 723 000. The vast majority of cases of the gastric cancer are sporadic, but it is known that 1-3\% are a manifestation of the hereditary forms and syndromes. This article is dedicated to the new trends and strategies in the management of the risk of the hereditary diffuse gastric cancer. The basics of genetic counseling and current criteria for selecting families are covered, in the aim of analyzing hereditary mutations. The problems of endoscopic screening and dynamic monitoring of the gastric mucosa are discussed. A possible approach of the surgical treatment as preventive gastrectomy is discussed. The features of morphological studies in hereditary diffuse gastric cancer are outlined. The principles of postoperative care are covered. The prospects of medical support for patients with hereditary diffuse stomach cancer are reviewed.

Keywords: hereditary gastric cancer; diffuse gastric cancer; CDH1 gene mutation; genetic counseling.

\section{Введение}

В структуре онкологической заболеваемости во всем мире рак желудка (РЖ) занимает пятое место и является третьей среди наиболее распространенных причин смерти от рака, абсолютное число смертельных случаев в год оценивается в 723000 [1]. Однако на настоящий момент не разработаны рекомендации по ведению пациентов с наследственным диффузным раком желудка, тематика наследственного рака желудка в отечественной медицинской литературе освещена недостаточно. Подавляющее большинство случаев РЖ имеют спорадический характер, но известно, что 1-3\% являются проявлением наследственных форм и синдромов. Так, к данным синдромам относятся: синдром Ли-Фраумени, синдром Линча, синдром Пейтца-Егерса, наследственный рак молочной железы (РМЖ) и яичников, ассоциированный с мутациями в генах BRCA1/2, MUTYH-ассоциированной полипоз, семейный аденоматозный полипоз (САП), ювенильный полипоз и синдром множественных гамартом, ассоциированный с геном PTEN (синдром Коудена). Риск развития рака желудка при этих синдромах существенно различается по данным различных популяционных исследований, но в целом низок. Корреляционный анализ, проведенный впервые более 15 лет назад установил, что наследственные мутации в гене $C D H 1$, кодирующем супрессорный белок Е-кадгерин, являются причиной развития наследственного диффузного рака желудка (НДРЖ) [2, с. 403]. В 1999 г. были установлены критерии для отбора семей с целью проведения скрининга наследственных мутаций в гене $C D H 1$, их пересмотр произошел в 2010 г. [3, с. 440-441]. Не все 
семьи, удовлетворяющие этим критериям, имеют мутации в гене $C D H 1$, что указывает на возможность вовлечения других генов в развитие предрасположенности к диффузному раку желудка (ДРЖ). Наследственные мутации гена CTNNA1 были описаны в трех семьях, у представителей которых был выявлен ДРЖ, и только одна из этих семей удовлетворяла критериям НДРЖ от 2010 г. [4, с. 30].

\section{Генетическое консультирование и анализ мутации}

Генетическое консультирование является важным компонентом оценки и управления риском при НДРЖ. Оно должно включать в себя стандартное генетическое обследование медицинским генетиком, сбор подробного семейного анамнеза, оценивающего не менее трех поколений родословной пробанда, гистологическое подтверждение диагноза ДРЖ и/или предраковых состояний (карцинома in situ или педжетоидное распределение перстневидных клеток), а также прогнозирование риска развития ДРЖ. Необходимо оформление добровольного информированного согласия на проведение генетического тестирования. К пациентам с мутацией в гене $C D H 1$ должен быть применен мультидисциплинарный подход, задействована многопрофильная команда специалистов (МПКС), имеющих опыт в абдоминальной хирургии, гастроэнтерологии, диетологии, патоморфологии, оказании психосоциальной поддержки. Генетическое тестирование может проводитсяс момента совершеннолетия пробанда (в среднем с возраста 16-18 лет). Тестирование более молодых членов семьи при отсутствии установленного у них диагноза может быть рассмотрено в индивидуальном порядке. Зарегистрированы случаи ДРЖ в возрасте до 18 лет у членов семей, генетически отягощенных по данному заболеванию [5, с. 354], однако в общем риск развития ДРЖ до 20 лет крайне низок [6, с. 1349].

По результатам недавнего исследования 75 пациентов с ДРЖ - представителей семей, отягощенных носительством мутации гена $C D H 1$, - обновлена информация по пенетрантности патогенной мутации гена $C D H 1$. Семьи с миссенс-мутацией в гене $C D H 1$, а также семьи, для которых информация о генетическом тестировании недоступна, были исключены из исследования. Совокупный риск ДРЖ у носителей мутации $C D H 1$ к возрасту 80 лет составил 70\% для мужчин (95\% ДИ 59-80\%) и $56 \%$ для женщин (95\% ДИ 44-69\%) [4, с.30].

Медицинские генетики (или другие члены МПКС) должны проинформировать консультируемого о перспективах операции на желудке и возможностях динамического наблюдения. Подтвержденным носителям патогенных 
мутаций $C D H 1$ рекомендуется профилактическая гастрэктомия. Некоторые пациенты могут пожелать отложить проведение профилактической гастрэктомии по тем или иным причинам, для них эндоскопическое динамическое наблюдение следует рассматривать как промежуточный этап перед оперативным лечением.

\section{Критерии для проведения тестирования наследственных мутаций гена $\mathrm{CDH} 1$}

Последние рекомендации 2010 г. расширили клинические критерии отбора пациентов для тестирования на мутации гена $C D H 1$.Частота выявления мутаций $C D H 1$ до 2010 г. с использованием критериев отбора, установленных в 1999 г., составляла 25-50\% [7, с. 22]. Использование новых критериев привело к снижению выявления случаев носителей мутаций гена CDH1 в странах с низким уровнем заболеваемости РЖ, которое снизилось до $10-18 \%$ [4, с. 30]. Это снижение можно частично объяснить обогащением крупных и подозрительных в отношении ДРЖ семей (то есть появлением новых членов семей, не являющихся носителями мутаций), которые вошли в исследования, опубликованные до 2010 г. Следует отметить, что настоящие критерии предназначены для стран с низким уровнем заболеваемости спорадическим РЖ. Для стран с высоким уровнем заболеваемости спорадическим РЖ имеются немногочисленные публикации или разборы отдельных клинических случаев пациентов с выявленной $C D H 1$ мутацией, однако крупные когортные исследования на настоящий момент отсутствуют. По данным немногочисленных публикаций из Южной Кореи, Японии и Португалии, уровень обнаружения патогенных наследственных мутаций $C D H 1$ при НДРЖ и раннем РЖ оценивается в 8-15\% [8, с.1898]. В рамках формирования рекомендаций, принятых в 2010 г., проведено объединение первого (два или более случаев РЖ в семье, один ДРЖ в возрасте моложе 50 лет) и второго (три или больше случаев ДРЖ независимо от возраста) критериев тестирования в один: два или более случаев РЖ независимо от возраста, по меньшей мере один из которых - подтвержденный гистологически ДРЖ у родственников первой и второй степеней родства. Два других критерия тестирования не были изменены: пачиент с ДРЖ моложе 40 лет с собственной или семейной историей ДРЖ и ДРМЖ, при этом одно из заболеваний возникло в возрасте до 50 лет. Есть и другие семьи, в которых необходимо проведение генетического тестирования. Нaличие двустороннего долькового рака молочной железы (ДРМЖ) в возрасте до 50 лет или наличие нескольких близких родственников с ДРМЖ (двое 
из которых в возрасте до 50 лет) определяет показание к тестированию на мутации CDH1 [9, с. 487]. Тестирование следует также проводить лицам, у которых имеется заячья губа и/или волчья пасть в сочетании с ДРЖ; данное положение также относится и к членам семей, где встречается это сочетание [10, с. 139-140]. В тех случаях, когда патоморфолог выявляет карциному in situ, представленную перстневидными клетками и/или педжетоидное распределение перстневидных клеток в слизистой оболочке желудке (СОЖ), также следует проводить генетическое тестирование, так как это редкое состояние крайне нехарактерно для спорадического рака. Генетическое тестирование следует, по возможности, проводить пробанду, у которого выявлены изменения. Если пробанд, у которого выявлены изменения, умер, но имеются замороженные, фиксированные формалином или парафином ткани (предпочтительно не пораженные опухолью), необходимо провести их тестирование на наследственные мутации гена $\mathrm{CDH1}$. В случае, если ткани больного отсутствуют или их качество недостаточно для выполнения теста, приемлемым является тестирование родственников. При этом тестирование должно быть выполнено, по меньшей мере, троим родственникам первой степени родства одновременно, тем самым увеличивается вероятность обнаружения мутации $C D H 1$. Следует помнить, что тестируемые родственники имеют 50\% вероятность наследования мутантного аллеля гена CDH1.

\section{Генетическое тестирование: лабораторные возможности}

Генетическое тестирование крови или ткани на наследственные мутации должно быть выполнено в сертифицированных молекулярных диагностических лабораториях. Анализ гена $C D H 1$ должен включать мутационный анализ всей открытой рамки считывания, включая границы интронов и экзонов, а также анализ числа копий отдельных экзонов для обнаружения внутригенной делеции или дупликации экзона. В настоящее время анализ в основном осуществляется с помощью комбинации секвенирования по Сенгеру и мультилокусного лигирования с последующей амплификацией (MLPA). На сегодняшний день описано более 155 различных наследственных мутаций гена $C D H 1$, большинство из которых являются патогенными, но имеется и ряд вариантов неопределенного значения [11, с. 365-366]. CDH1 представляет собой ген-супрессор опухоли и, следовательно, необходимы изменения во втором аллеле для инициации опухолевого процесса. Пусковой механизм и молекулярный механизм, с помощью которого второй аллель $C D H 1$ инактивируется, может включать в себя метилирование, соматические мутации и потерю гетерозиготности [12, с. 300]. К настоящему времени Институ- 
том молекулярной патологии и иммунологии университета Порту (Португалия) зарегистрировано 49 наследственных вариантов миссенс-мутаций гена $C D H 1$, большая часть которых классифицируется как вредные и, таким образом, возможно, они являются патогенными.

В настоящее время не существует международной базы данных, которая содержит все наследственные мутации $C D H 1$ и полиморфные варианты, выявленные на текущий момент. Эта база в настоящее время находится в стадии формирования http://www.LOVD.nl/CDH1. Она может быть использована с целью уточнения, является ли выявленная мутация $C D H 1$ уже известной, ведет ли она к развитию заболевания или возникает по его причине, выявлена ли она популяционным анализом, сегрегационным анализом, получена в моделируемом эксперименте, функциональным анализом в invitro или данные изменения редки и встречаются у ограниченного числа индивидов, семей.

В настоящее время описаны три семьи, отвечающие новым принятым критериям, у них выявлена наследственная мутация гена CTNNA1 [4, с. 30]. Положение о наличии в семье $C D H 1$ мутации не является обоснованным, даже если клиническая картина заболевания сходна с той, что есть в семьях носителей $C D H 1$ мутации. С учетом функциональной связи между двумя генами они могут представлять собой лишь копию данного гена. Носители таких мутаций должны быть проинформированы о возможности проведения профилактической гастрэктомии и других мер по профилактике рака, рекомендованных для НДРЖ, но с той оговоркой, что данные рекомендации не имеют под собой достаточного количества научных исследований. Недавно были описаны семьи с мутациями генов $B R C A 2$ и $P A L B 2$, к ним рекомендуется применять те же принципы, что описаны в национальных руководствах по НДРЖ. Вполне вероятно, что будут открыты мутации других генов, ассоциированные с НДРЖ, с использованием секвенирования следующего поколения. Так, в результате использования разных методов анализа генома была описана мутация гена MAP3K6 [13].

\section{Скрининг и динамическое наблюдение}

\section{Эндоскопический скрининг и динамическое наблюдение} за состоянием слизистой оболочки желудка

Для пациентов с неопределённым статусом мутации $C D H 1$ эндоскопическое исследование является скринингом, в то время как для пациентов с подтвержденной мутацией гена данное исследование проводится в рамках динамического наблюдения. Индивидам, у которых выявлена 
наследственная мутация $C D H 1$, должна быть рекомендована профилактическая гастрэктомия, независимо от находок, выявленных при эндоскопическом исследовании. Тем не менее, сроки проведения операции могут варьировать в зависимости от собственного решения пациента, возраста, а также его физической и психологической готовности. Пациентам, которым планируется гастрэктомия, должно быть выполнено предоперационное эндоскопическое исследование с целью поиска макроскопических фокусов опухоли, так как это может привести к изменению плана лечения. Эндоскопическое исследование также выполняется, чтобы исключить другие заболевания, например, пищевод Барретта, которые могут изменять объем оперативного вмешательства.

Пациентам с мутацией $C D H 1$, которым не была проведена гастрэктомия по причине отказа пациента или наличия противопоказаний по физическому или психическому состоянию, следует проводить регулярные эндоскопические исследования. Пациентам, отказывающимся от хирургического вмешательства, динамическое наблюдение может помочь в принятии решения о необходимости гастрэктомиив случае выявления у них микроскопических очагов опухоли или перстневидных клеток. Тем не менее, пациенты должны быть осведомлены, что отсрочка операции может быть крайне опасным решением [14, с. 80-81].

Ведение лиц с результатом тестирования на мутацию $C D H 1$ «неопределенного значения» и тех, в чьей семье может быть выявлена мутация данного гена, является непростой задачей. Мы рекомендуем интенсивное динамическое эндоскопическое наблюдение в экспертном центре семьям, которые удовлетворяют критериям НДРЖ. Эндоскопический скрининг играет важную роль в принятии клинических решений, так как у 2/7 пациентов (1/5 семей), удовлетворяющих критериям НДРЖ, получают отрицательный результат тестирования на мутации $C D H 1$ [14, с 80]. Так, пациентам, у которых эндоскопически выявлены минимальные злокачественные поражения, все же следует рекомедовать гастрэктомию. Все пациенты, удовлетворяющие критериям НДРЖ, прошедшие эндоскопическое исследование, должны быть проинформированы, что, учитывая мелкоочаговый характер изменений и их плохую эндоскопическую визуализацию, данные изменения не могут быть подтверждены результатами неприцельной биопсии.

\section{Эндоскопический протокол НДРЖ}

Эндоскопические исследования следует выполнять в центрах, где есть мультидисциплинарная база. Тем не менее, нужно понимать, что часть па- 
циентов не смогут добраться до таких центров по тем или иным причинам. В этом случае консультация экспертного центра по протоколу эндоскопического исследования, проведенного по месту проживания, в комбинации с гистологическим исследованием могут стать хорошей альтернативой. Как было отмечено выше, оптимальная частота проведения эндоскопического исследования не определена. На основе имеющегося опыта рекомендуется ежегодное проведение эндоспического исследования. При этом риск кровотечения может быть несколько выше ввиду большего числа биопсий. В этой связи рекомендуется отменять антикоагулянты накануне процедуры. Эндоскопическое исследование должно быть выполнено с использованием эндоскопа высокой четкости (HD), в белом свете, процедура должна занимать не менее 30 минут, чтобы обеспечить тщательный осмотр слизистой оболочки при многократном раздувании и сдувании желудка, а также сопровождаться сбором биоптатов. СОЖ должна быть тщательно отмыта перед исследованием комбинацией муколитика и пеногасителя, смешанных со стерильной водой. Макроскопическая картина СОЖ и любые очаговые изменения должны быть сфотографированы или фиксированы на видео для дальнейшего соотношения с результатами гистологического исследования образцов, полученных при биопсии.

До начала оценки мелких очагов желудок должен быть неоднократно раздут и сдут с целью оценки его растяжимости. Плохая растяжимость стенки желудка, отсутствие расправления складок должны рассматриваться как возможный инфильтративный рост опухоли, как linitis plastica. Если заподозрен подслизистый рост опухоли, помимо биопсии следует проводить мультиспиральную компьютерную томографию и эндоскопическое ультразвуковое исследование с целью визуализации слоев стенки желудка.

Из-за того, что очаги перстневидных клеток могут быть крайне малы и определены только при микроскопии, необходимо проводить многократную биопсию (с получением большого числа биоптатов), чтобы увеличить вероятность морфолической верификации диагноза [12, с. 295]. Рекомендуется проводить биопсию любых эндоскопически видимых участков поражения, в том числе и бледных участков слизистой. Следует проводить биопсию с забором материала слизистой каждой из следующих анатомических зон: препипилорического, антрального отделов, переходной зоны, тела, дна и кардии. По Кембриджскому протоколу необходим забор не менее 30 образцов ткани [3, с. 437], даже если это будет приводить к увеличению выборки из-за большой площади слизистой желудка, хотя забор большего числа биоптатов на практике технически не выполним [15, с. 1710]. Биопсия может быть сделана с использованием стандартных щип- 
цов, в идеале оборудованных шипом, так как это поможет захватить собственную пластинку слизистой, в которой могут присутствовать очаги перстневидных клеток. В случае четко визуализируемого поражения целесообразно выполнять эндоскопическую резекцию слизистой оболочки для получения адекватного образца ткани, гистологическое исследование которого позволит оценить и задокументировать степень инвазии. Данную манипуляцию следует рассматривать как диагностическую, а не лечебную, ввиду мультифокальной характера поражения.

Особое внимание необходимо уделять бледным зонам СОЖ, так как они могут содержать микроскопические скопления атипичных клеток, не являясь специфичными очагами [16, с. 465]. Последние данные также свидетельствуют о том, что данные области видны при тщательном рассмотрении в белом свете, а также с использованием NBI-режима (режим, позволяющий оценивать сосудистые структуры), что позволяет лучше оценивать данные зоны.

\section{Будущее динамического наблюдения за состоянием}

\section{слизистой желудка}

В свете появления новых эндоскопических технологий, таких как NBI-режим, BLI-режим, I-scan режим, аутофлуоресцентная эндоскопия, $\mathrm{IHb}$-усиление и конфокальная прижизненная эндомикроскопия, значительно улучшающих диагностику, необходимо продолжение исследований их возможного применения с целью оптимизации эндоскопического мониторинга лиц с высоким риском развития ДРЖ. Учитывая редкость этого состояния, должны быть проведены многоцентровые исследования, придерживающиеся строго определенного протокола. Кроме того, следует отметить, что протокол мультифокальной биопсии приводит к образованию рубцов, которые в дальнейшем могут принимать вид бледных пятен. Также было бы очень ценно сравнить эффективность подхода прицельной биопсии с текущим Кембриджским протоколом, который предполагает проведение нескольких случайных биопсий. Такие исследования обобщат данные об изменениях, наблюдаемых в бледных областях, и помогут определить особенности, указывающие на фокусы перстневидных клеток.

\section{Гастрэктомия}

\section{Профилактическая гастрэктомия: показания и сроки проведения операции}

Профилактическую гастрэктомию следует рекомендовать подтвержденным носителям патогенной герминальной мутации $C D H 1$. Некото- 
рые авторы утверждают, что термин «профилактическая гастрэктомия» является неточным и апеллируют к термину «риск-снижающая гастрэктомия», поскольку большинство носителей мутации к моменту операции уже имеют микроскопические очаги перстневидных клеток. Для этих пациентов гастрэктомия полностью исключает риск развития карциномы желудка и действительно является профилактической с точки зрения предотвращения смерти от рака желудка.

Оптимальные сроки проведения профилактической гастрэктомии неизвестны, и, как правило, весьма индивидуальны. Так как эта операция в значительной степени влияет на качество жизни, решение о ней должно быть принято взвешенно, объективно и своевременно. Важное значение имеет консультирование по поводу плюсов и минусов вмешательства. Обсуждение возможности проведения гастрэктомии с носителями наследственной мутации $C D H 1$ должно проводиться по достижении ими возраста 20-30 лет $[17$, c. 270$]$. Решение о проведении профилактической гастрэктомии носителям мутации CDH1 старше 75 лет должно приниматься на основании информации о физическом состоянии пациента и риске развития осложнений. У пробандов следует принимать во внимание семейный фенотип, возраст наступления клинических проявлений рака. Вероятно, существует период, в течение которого перстневидные клетки не распространяются или не наблюдается их прогрессивного размножения, пока пролиферативный индекс низкий, поэтому очевидно, что сроки выполнения профилактической гастрэктомии должны быть раньше клинических проявлений рака [12, c. 301-302]. Это объясняет, почему после профилактической гастрэктомии опухоли стадируют как T1N0 [18, с. 875]. Пациенты, имеющие клинические проявления распространенного НДРЖ имеют плохой прогноз, и лишь 10\% имеют потенциальную возможность к излечению [19, с. 347]. Даже если лечение оценивается как потенциально возможное, 5-летняя выживаемость по-прежнему не превышает $30 \%$ [20, с. 687]. И пока наши знания о патогенезе перстенвидноклеточного рака накапливаются, у некоторых пациентов есть возможность отложить профилактическую гастрэктомию до половозрелого возраста, когда ее проведение более безопасно. Важно долгосрочное мультидисциплинарное наблюдениеза пациентами, которым проведена профилактическая гастрэктомия на протяжении всей их жизни.

\section{Особенности операции}

Необходимый объем операции состоит в гастрэктомии с реконструктивным анастомозом по Ру таким образом, чтобы тонкокишеч- 
но-тонкокишечный анастомоз находился на расстоянии не менее $50 \mathrm{~cm}$ дистальнее пищеводно-желудочного с целью уменьшения рискабилиарного рефлюкса. Проксимальная линия резекции должна проходить через дистальный отдел пищевода, слизистая которого представлена многослойным плоским неороговевающим эпителием, чтобы гарантировать отсутствие слизистой кардиального отдела в оставшейся проксимальной части [21, с. 145]. Это может быть подтверждено путем срочного гистологического исследования проксимального края резекции или эндоскопической маркировки зоны пищеводно-желудочного перехода на операционном столе.

Оптимальный объем лимфодиссекции пока обсуждается. Среди пациентов с ранней стадией аденокарциномы желудка кишечного типа (pT1a) частота метастазирования в лимфоузлы составляет $2-5 \%$ [22, с. 277] и увеличивается до 6\% при недифференцированном или диффузном типе $[23$, c. 510$]$. В опухолях pT1b c инвазией в подслизистый слой метастазы в лимфатические узлы обнаруживаются в 17-28\%, увеличиваясь в процентном соотношении в зависимости от глубины инвазии. У большинства пациентов, проходящих профилактическую гастрэктомию по поводу НДРЖ, выявляется по крайней мере Т1а стадия распространения опухоли. Поскольку предоперационная гастродуоденоскопия не может исключить наличие T1b степени поражения и, соответственно, более высокого риска метастатического поражения лимфоузлов, а в ходе интраоперационной ревизии также не удается полноценно оценить статус опухолевого поражени лимфатических узлов, то проведение лимфодиисекции, как минимум в объеме D1 (с включением лимфатических узлов групп 1-7) является оправданным шагом.

\section{Морфология}

\section{Гистологическое исследование биоптатов жслудка пациентов,} у которых заподозрен НДРЖ

Биоптаты пациентов с отягощенным семейным анамнезом по ДРЖ, которые прошли эндоскопический скрининг в соответствии с Кембриджским протоколом, следует тщательно исследовать. Биоптаты должны быть окрашены гемотакслином и эозином, а также по Шиффу, что является стандартом исследования. Специфическое поражение при НДРЖ, представленное мелкими очагами типичных перстневидных клеток, иногда может быть дополнено наличием небольшого числа мелких атипичных 
клеток в собственной пластинке слизистой оболочки без инфильтрации подслизистой. Преинвазивные поражения при перстневидноклеточном раке (ПКР) могут быть представлены:

1) ПРК in situ, что соответствует наличию перстневидных клеток с гиперхромными и деполяризованными ядрами на базальной мембране желез, замещающих нормальные клетки этой железы;

2) педжетоидным распределением перстневидных клеток под сохранившимся эпителием желез и ямок, а также в базальной мембране [24, с. 60].

\section{Гистологическое исследование при гастрэктомии: классификация} микроскопических очагов и определение роли их выявления

Макроскопическая оценка и отбор проб из препарата после профилактических гастрэктомий должны следовать протоколу. Гистологическое описание должно быть сделано с использованием контрольного списка, представленного в таблице 1.

\section{Оценка морфологических изменений СОЖ}

Таблиия 1.

\begin{tabular}{|l|l|}
\hline \multicolumn{2}{|c|}{ Контрольный список для оценки морфологического материала прилученного } \\
\multicolumn{1}{|l|}{$\begin{array}{l}\text { при профилактической гастрэктомии } \\
\text { для очагов } \geq \mathrm{p} T 1 \mathrm{~b}\end{array}$} & $\begin{array}{l}\text { Паттерн роста (диффузная инфильтрация против локазованной } \\
\text { опухоли) } \\
\text { Анатомическая локализация (кардия, дно, тело, переходная зона, } \\
\text { антральный отдел) } \\
\text { Размеры очага } \\
\text { Гистологический тип в соответсвии с классификацией ВОЗ [25] и } \\
\text { Лорена [26] } \\
\text { Инвазия в лимфатические, венозные сосуды и нервы (наличие, от- } \\
\text { сутствие) } \\
\text { Опухоль, лимфатические узлы, отдаленные метастазы }\end{array}$ \\
\hline $\begin{array}{l}\text { 2. Характеристики } \\
\text { для очагов рТ1а } \\
\text { ПКР }\end{array}$ & $\begin{array}{l}\text { Количество очагов } \\
\text { Анатомическая локализация (кардия, дно, тело, переходная зона, } \\
\text { антральный отдел) } \\
\text { Размеры очага } \\
\text { Агрессивные черты опухоли: полимофизм, потеря муцина, шпин- } \\
\text { дель клетки, мелкие клетки, митозы } \\
\text { Стромальная реакция, связанная с опухолью: десмопластическая, } \\
\text { лимфатическая, эозинофильная или гранулематозная воспалитель- } \\
\text { ная реакция } \\
\text { Края резекции (проксимальный пищеводный, дистальный - сли- } \\
\text { зистая двенадцатиперстной кишки, включая луковицу), с целью } \\
\text { подтверждения удаления всей слизистой желудка и отсутствя опу- } \\
\text { холевой ткани в краях резекции } \\
\text { Состояниелимфатических узлов }\end{array}$ \\
\hline
\end{tabular}


Окончание табл. 1.

\begin{tabular}{|c|c|}
\hline $\begin{array}{l}\text { 3. Изменения } \\
\text { слизистой не от- } \\
\text { носящиеся к нео- } \\
\text { плестическим: из- } \\
\text { менения, которые } \\
\text { наиболее часто } \\
\text { встречаются при } \\
\text { данных случаях }\end{array}$ & $\begin{array}{l}\text { Гиперпластические изменения слизистой } \\
\text { Вакуолизация поверхностного эпителия } \\
\text { Сферические изменения }\end{array}$ \\
\hline $\begin{array}{l}\text { 4. Другие находки } \\
\text { в окружающей } \\
\text { слизистой }\end{array}$ & $\begin{array}{l}\text { Воспалительные изменения (острое, хроническое, эрозия, изъязвле- } \\
\text { ние) } \\
\text { Н.руlогі } \\
\text { Внутриэпителиальные лимфоциты } \\
\text { Лимфоидная инфильтрация } \\
\text { Железистая атрофия } \\
\text { Кишечная метаплазия } \\
\text { Аденоматозная дисплазия }\end{array}$ \\
\hline
\end{tabular}

Практически во всех препаратах, полученных после гастрэктомий, были выявлены мелкие очаги ПКР или рак insitu из перстневидных клеток, хотя иногда они были обнаружены только после пересмотра морфологом, специализирующимся в данной области $[5$, с. $355 ; 27$, с. $348 ; 28,26]$.

Наличие большого числа очагов аденокарциномы с градацией Т1а и малого числа с градацией in situ предполагает, что инвазия в собственную пластинку слизистой перстневидных клеток может возникать без наличия морфологически подтвержденной карциномы in situ [29, с. 26]. Очень важно маркировать участки забора биоптатов в препарате удаленного желудка, чтобы узнать больше о механизмах распространения раннего НДРЖ.

Обязательно следует оценивать края резекции на предмет наличия резидуальной слизистой желудка и опухоли. Следует отметить, что железы кардиального типа могут диффузно располагаться в собственной пластинке слизистой на разных участках пищевода. Риск для развития ПКР в железах кардиального отдела желудка не определен, частота его развития не оценена статистически [30]. Тем не менее, метапластическая и гетеротопная слизистая желудка может определяться на различных участках ЖКТ. Так, в группе из 19 пациентов, которым была проведена гастрэктомия, в трех случаях (16\%) была выявлена гетеротопическая слизистая желудка в области двенадцатиперстной кишки, и в одном из трех (5,3\%) был выявлен ПКР [31].

\section{Гистологическое исследование при распространенном НДРЖ}

Как и спорадический ДРЖ, распространенный НДРЖ преимущественно представлен в виде linitis plastica с диффузной инфильтрацией стенки 
желудка. При гистологическом исследовании определяются сгруппированные или единичные перстневидные клетки. Обычно данные опухоли состоят из полиморфного неопластического инфильтрата с небольшим количеством или полным отсутствием классических перстневидных клеток. В меньшем количестве случаев опухолевые клетки располагаются в небольших агрегатах, иногда розетками или железоподобными структурами. Также могут выявляться скопления внеклеточного муцина, в котором плавают опухолевые клетки. Хотя нет никаких специфических морфологических признаков, указывающих на наследственный характер опухоли, поражения in situ и педжетоидное распространение перстневидных клеток в окружающую нормальную слизистую являются важными характеристиками для постановки диагноза. Анализ 103 образцов желудка у членов семей с НДРЖ, не имеющих установленной наследственной мутации гена $C D H 1$, показал сходную морфологическую картину, характерную для носителей наследственной мутации гена $C D H 1[32]$.

\section{Использование иммуногистохимических исследований (ИГХИ)}

Местно-распространенный ДРЖ с обширной инвазией может быть легко обнаружен при окрашивании гематоксилином и эозином. Использование ИГХИ для нейтрального муцина, окраска Шифф-йодной кислотой с предварительной обработкой диастазой целесообразны для обнаружения или подтверждения мелких внутрислизистых очагов, в которых опухолевые клетки распределены среди сохранившихся ямок и желез. Данные виды окрашивания должны выполняться рутинно при обработке образцов полученных при биопсии желудка в ходе эзофагогастродуоденоскопии (ЭГДС) и пригастрэктомии, выполненных пациентам с НДРЖ [33, с. 1008]. Окраска на цитокератины может помочь подтвердить эпителиальную природу перстневидных клеток, если есть какие-либо сомнения. Иммуноэкспрессия Е-кадгерина может быть снижена или отсутствовать при раннем раке, контрастирование в норме присутствующего в мембране Е-кадгерина может наблюдается в граничащей с опухолью интактной СОЖ [34, с. 2137]. При педжетоидном распространениии карциноме in situ иммуноэкспрессия Е-кадгерина также может быть снижена или отсутствовать. Однако экспрессия Е-кадгерина не всегда снижается или отсутствует; все зависит от типа мутациии специфических механизмов инактивации аллеля дикого типа [34, с. 2137]. При ДРЖ у пациентов, не имеющих наследственной мутаций гена CDH1, паттерн экспрессии Е-кадгерина также часто снижается или отсутствует. Таким образом, окрашивание Е-кадгерина не следует ис- 
пользовать в качестве способа предварительного скрининга для отбора пациентов, имеющих наследственную мутацию CDH1.

\section{Перспектива исследований в области молекулярного патогенеза НДРЖ}

Вопрос, на который пока нет ответа, как долго ранний НДРЖ не будет проявляться клинически? На каком этапе развития заболевания пациенту потребуется экстренная помощь, опухоль станет быстро прогрессировать, когда приведет к летальному исходу?

Продолжение сбора данных о пациентах, которые выбирают консервативную тактику динамического эндоскопического наблюдения, имеет важное значение и поможет ответить на этот вопрос. Кроме того, необходим тщательный анализ механизмов, ответственных за инактивацию второго аллеля гена $C D H 1$ как самого раннего изменения, приводящего к развитию НДРЖ, что впоследствии поможет определить стратегии для проведения химиопрофилактики. Молекулярный фон больных с НДРЖ в отсутствии выявленной мутации гена $C D H 1$ еще предстоит уточнить, в том числе необходимо отслеживать любые специфические морфологические особенности РЖ с целью установления других наследственных синдромов рака. Маевский $[35$, с. 622-624] описал семью, в которой выявлена наследственная мутация гена CTNNA1, приводящая к развитию ДРЖ, представленного множественными внутрислизистыми очагами перстневидных клеток. ИГХИ на альфа-Е-катенин, направленное на выявление перстневидных клеток, не дали окрашивания, в то время как опухоли из 10 других родословных, имеющих НДРЖ, но не имеющих мутации генов CDH1 и CTNNA1, при данном окрашивании выявлялись. Недавно были описаны две семьи, одна из которых имела иммуногистохимический фенотип с потерей альфа-катенина и Е-кадгерином в большей степени представленным в цитоплазме, а не на мембране клетки [4, с. 31]. Этот факт позволяет предположить, что либо патогенность мутации гена CTNNA1 опосредована через потерю функции Е-кадгерина, либо рак-инициирующий потенциал мутации гена CDH1 peaлизуется через альфа-катенин-ассоциированный путь.

\section{Послеоперационный уход и диета}

\section{Ведение послеоперационного периода}

Психологические, физиологические и метаболические последствия гастрэктомии не следует недооценивать. Нарушение физиологических 
процессов для конкретного индивида предсказать трудно, но есть надежда, что большинство пациентов вернутся к полноценной и активной жизни после операции.

Уровень качества жизни возвращается к предоперационному состоянию через 12 месяцев; тем не менее, проблемы с приемом пищи, боли в животе и дефицит массы тела в эти сроки не разрешаются [36, с. 88]. В настоящее время существует множество программ восстановления после гастрэктомии. Эти программы могут включать в себя предоперационную углеводную нагрузку, отказ от дренирования желудка и брюшной полости, раннюю активация в комбинации с адекватной анальгезией (эпидуральная или регионарная анестезия), возобновление питания через рот в течение 3-5 дней и выписку из стационара в течение 7-10 дней. Кроме того, пациенты в течение первых 12 месяцев после операции нуждаются в посторонней помощи.

\section{Диета}

Основные моменты, которые должны быть корригированы диетой и режимом питания после гастрэктомии:

1) поддержание массы тела;

2) обеспечение необходимой водной нагрузки, поступления достаточного количества питательных веществ, достижение должного калоража;

3) изменение привычек, связанных с приемом пищи.

Средняя потеря массы тела после гастрэктомии составляет 10 кг в год [36, с. 88]. Это означает, что пациенты, которые уже имели недостаток массы тела до операции или нарушение пищеварения, нуждаются в консультации и помощи.

Изменение анатомии желудочно-кишечного тракта (ЖКТ) может нарушить акт приема пищи, что в свою очередь приведет к отсутствию удовольствия от приема пищи и психологическому нарушению акта приема пищи, а соответственно невозможности контроля массы тела. В раннем послеоперационном периоде насильственный прием пищи и жидкости может затруднить восстановление. Пациенту важно понимать перспективу выздоровления и то, что его состояние со временем улучшится.

После гастрэктомии пациенты должны принимать пищу малыми порциями. Прием больших объемов пищи и/или слишком быстрый прием пищи будет вызывать боли в животе. Демпинг-синдром представляет собой группу симптомов, которые возникают в результате изменения анатомии ЖКТ после гастрэктомии. Они обусловлены быстрым поступлением пищи в тонкую кишку и наступлением кишечной стадии пищеварения, 
минуя желудочную, что в свою очередь приводит к переходу жидкости из сосудистого русла в тонкую кишкус целью разжижения химуса, что вызывает различные симптомы со стороны сердечно-сосудистой системы и органов брюшной полости. Поздний демпинг-синдром вызван быстрым ростом и последующим снижением уровня сахара в крови за счет запоздалой секреции инсулина. После резекции желудка пища сразу попадаетв тонкую кишку, где онабыстро переваривается. Кроме того, поджелудочная железа вырабатывает больше инсулина за короткий временной промежуток и его избыток приводит к быстрому снижению уровня глюкозы крови [37, с. 629-630]. Эти симптомы часто возникают в раннем послеоперационном периоде и постепенно регрессируют с течением времени. Проявления демпинг-синдрома могут быть сведены к минимуму или устраненыпутем подбора диеты и изменения пищевых предпочтений; в каждом отдельном случае проводимая коррекция строго индивидуальна для каждого пациента.

Другие нарушения могут включать в себя непереносимость лактозы, стеаторею, рост бактерий в тонкой кишке, стриктуру анастомоза, ощущение переполнения желудка [38, с. 191]. Следует информировать пациента о том, что толерантность к алкоголю после гастрэктомии снизится, а также может потребоваться изменение дозы ранее принимаемых препаратов.

После гастрэктомии в результате мальабсорбции может возникать дефицит питательных веществ. Мониторинг биохимических показателей и оценка питания необходимы как в раннем послеоперационном периоде, так и в отдаленные сроки, так как недостатки отдельных элементов увеличивают риск возникновения других симптомов и заболеваний. Все пациенты должны пожизненно получать витамин В12 (пероральное, подкожное или внутримышечно) для компенсации его недостатка, контролировать уровень железа с целью профилактики анемии, уровень кальция с целью профилактики остеопороза, а также контролировать уровни микроэлементов с целью профилактики дисмикроэлементоментозов. Многие диетологи рекомендуют пациентам ежедневно принимать мультивитаминные комплексы с железом, однако контролировать уровень железа необходимо, так как вследствии гатрэктомии может измениться его усвоение. Также следует уделять внимание и другим неспецифическим симптомам, тем более, если они быстро не регрессируют, например, выпадение волос или сильная усталость. Эти симптомы и изменения могут быть признаком дефицита питательных веществ, который нужно выявить и разрешить. 


\section{Сопровождение пациента и последующие шаги} к получению медицинской помощи и исследования НДРЖ

Учитывая редкий характер НДРЖ пока не существует единой службы генетического консультирования, психологической поддержки, гастроэнтерологического ведения, хирургического обеспечения, морфологической диагностики и послеоперационного наблюдения семей ДНРЖ. География существующих экспертных центров не охватывает тех территорий, где необходима данная помощь. С новой тенденцией к развитию телемедицины, а также возникновению различных социальных групп пациентов, как 'No Stomach For Cancer' (http://www.nostomachforcancer. org/), 'De Gregorio Family Foundation for Stomachand Esophageal Cancer Research' (http://www.degregorio.org/) в США и 'Stichting CDH1' (http:// stichting-cdh1.nl/) в Нидерландах, очевиден имеющийся потенциал для сотрудничества между отдельными пациентами, медицинскими работниками, социальными объединениями пациентов с целью расширения возможностей диагностики и лечения пациентов с НДРЖ.

\section{Список литературы / References}

1. Ferlay J., Soerjomataram I., Ervik M., Dikshit R., Eser S., Mathers C., Rebelo M., Parkin D.M., Forman D., Bray F. GLOBOCAN 2012 v1.0, Cancer Incidence and Mortality Worldwide: IARC CancerBase No. 11 [Internet]. Lyon, France: International Agency for Research on Cancer; 2013. Available from: http://globocan.iarc.fr, accessed on day/month/year.

2. Guilford P., Hopkins J., Harraway J., McLeod M., McLeod N., Harawira P., Taite H., Scoular R., Miller A., Reeve A.E. E-cadherin germline mutations in familial gastric cancer. Nature, 1998, vol. 392, no. 6674, pp. 402-405.

3. Fitzgerald R.C., Hardwick R., Huntsman D., Carneiro F., Guilford P., Blair V., Chung D.C., Norton J., Ragunath K., Van Krieken J.H., Dwerryhouse S., Caldas C. Hereditary diffuse gastric cancer: updated consensus guidelines for clinical management and directions for future research. Journal of Medical Genetics, 2010, vol. 47, no. 7, pp. 436-444.

4. Hansford S., Kaurah P., Li-Chang H., Woo M., Senz J., Pinheiro H., Schrader K.A., Schaeffer D.F., Shumansky K., Zogopoulos G., Almeida Santos T., Claro I., Carvalho J., Nielsen C., Padilla S., Lum A., Talhouk A., Baker-Lange K., Richardson S., Lewis I., Lindor N.M., Pennell E., MacMillan A., Fernandez B., Keller G., Lynch H., Shah S.P., Guilford P., Gallinger S., Corso G., Roviello F., Caldas C., Oliveria C., Pharoah P.D.P., Huntsman D.G. Hereditary diffuse gastric cancer syndrome: CDH1 mutations and beyond. JAMA Oncology, 2015, vol. 1, no. 1, pp. 23-32. 
5. Wickremeratne T., Lee C.H., Kirk J., Charlton A., Thomas G., Gaskin K.J. Prophylactic gastrectomy in a 16-year-old. European Journal of Gastroenterology and Hepatology, 2014, vol. 26, no. 3, pp. 353-356.

6. Pharoah P.D., Guilford P., Caldas C. International Gastric Cancer Linkage C. Incidence of gastric cancer and breast cancer in CDH1 (E-cadherin) mutation carriers from hereditary diffuse gastric cancer families. Gastroenterology, 2001, vol. 121, no. 6, pp. 1348-1353.

7. Oliveira C., Seruca R., Carneiro F. Genetics, pathology, and clinics of familial gastric cancer. International Journal of Surgical Pathology, 2006, vol. 14, no. 1, pp. 21-33.

8. Oliveira C., Ferreira P., Nabais S., Campos L., Ferreira A., Cirnes L., Alves C.C., Veiga I., Fragoso M., Regateiro F., Dias L.M., Moreira H., Suriano G., Machado J.C., Lopes C., Castedo S., Carneiro F., Seruca R. E-Cadherin (CDH1) and 533 rather than SMAD4 and Caspase-10 germline mutations contribute to genetic predisposition in Portuguese gastric cancer patients. European Journal of Cancer, 2004, vol. 40, no. 12, pp. 1897-1903.

9. Benusiglio P.R., Malka D., Rouleau E., De Pauw A., Buecher B., Nogues C., Fourme E., Colas C., Coulet F., Warcoin M., Grandjouan S., Sezeur A., Laurent-Puig P., Moliere D., Tlemsani C., Di Maria M., Byrde V., Delaloge S., Blayau M., Caron O. CDH1 germline mutations and the hereditary diffuse gastric and lobular breast cancer syndrome: a multicenter study. Journal of Medical Genetics, 2013, vol. 50, no.7, pp. 486-489.

10. Frebourg T., Oliveira C., Hochain P., Karam R., Manouvrier S., Graziadio C., Vekemans M., Hartmann A., Baert-Desurmont S., Alexandre C., Lejeune Dumoulin S., Marroni C., Martin C., Castedo S., Lovett M., Winston J., Machado J.C., Attie T., Jabs E.W., Cai J., Pellerin P., Triboulet J.P., Scotte M., Le Pessot F., Hedouin A., Carneiro F., Blayau M., Seruca R. Cleft lip/palate and $\mathrm{CDH} 1 / \mathrm{E}-\mathrm{cadh}$ rin mutations in families with hereditary diffuse gastric cancer. Journal of Medical Genetics, 2006, vol. 43, no. 2, pp. 138-142.

11. Van der Post R.S., Vogelaar I.P., Carneiro F., Guilford P., Huntsman D., Hoogerbrugge N., Caldas C., Schreiber K.E., Hardwick R.H., Ausems M.G., Bardram L., Benusiglio P.R., Bisseling T.M., Blair V., Bleiker E., Boussioutas A., Cats A., Coit D., DeGregorio L., Figueiredo J., Ford J.M., Heijkoop E., Hermens R., Humar B., Kaurah P., Keller G., Lai J., Ligtenberg M.J., O’Donovan M., Oliveira C., Pinheiro H., Ragunath K., Rasenberg E., Richardson S., Roviello F., Schackert H., Seruca R., Taylor A., Ter Huurne A., Tischkowitz M., Joe S.T., van Dijck B., van Grieken N.C., van Hillegersberg R., van Sandick J.W., Vehof R., van Krieken J.H., Fitzgerald R.C. Hereditary diffuse gastric cancer: updat- 
ed clinical guidelines with an emphasis on germline $\mathrm{CDH} 1$ mutation carriers. Journal of Medical Genetics, 2015, vol. 52, no. 6, pp. 361-374.

12. Barber M., Murrell A., Ito Y., Maia A.T., Hyland .S, Oliveira C., Save V., Carneiro F., Paterson A.L., Grehan N., Dwerryhouse S., Lao-Sirieix P., Caldas C., Fitzgerald R.C. Mechanisms and sequelae of E-cadherin silencing in hereditary diffuse gastric cancer. Journal of Pathology, 2008, vol. 216, no. 3, pp. 295-306.

13. Gaston D., Hansford S., Oliveira C., Nightingale M., Pinheiro H., Macgillivray C., Kaurah P., Rideout A.L., Steele P., Soares G., Huang W.Y., Whitehouse S., Blowers S., LeBlanc M.A., Jiang H., Greer W., Samuels M.E., Orr A., Fernandez C.V., Majewski J., Ludman M., Dyack S., Penney L.S., McMaster C.R., Huntsman D., Bedard K. Germline mutations in MAP3K6 are associated with familial gastric cancer. PLOS Genetics, 2014, vol. 10, no. 10.

14. Lim Y.C., di Pietro M., O’Donovan M., Richardson S., Debiram I., Dwerryhouse S., Hardwick R.H., Tischkowitz M., Caldas C., Ragunath K., Fitzgerald R.C. Prospective cohort study assessing outcomes of patients from families fulfilling criteria for hereditary diffuse gastric cancer undergoing endoscopic surveillance. Gastrointestinal Endoscopy, 2014, vol. 80, no. 1, pp. 78-87.

15. Fujita H., Lennerz J.K., Chung D.C., Patel D., Deshpande V., Yoon S.S., Lauwers G.Y. Endoscopic surveillance of patients with hereditary diffuse gastric cancer: biopsy recommendations after topographic distribution of cancer foci in a series of $10 \mathrm{CDH} 1$-mutated gastrectomies. American Journal of Surgical Pathology, 2012, vol. 36, no. 11, pp. 1709-1717.

16. Shaw D., Blair V., Framp A., Harawira P., McLeod M., Guilford P., Parry S., Charlton A., Martin I. Chromoendoscopic surveillance in hereditary diffuse gastric cancer: an alternative to prophylactic gastrectomy? Gut, 2005, vol. 54, no. 4, pp. 461-468.

17. Blair V., Martin I., Shaw D., Winship I., Kerr D., Arnold J., Harawira P., McLeod M., Parry S., Charlton A., Findlay M., Cox B., Humar B., More H., Guilford P. Hereditary diffuse gastric cancer: diagnosis and management. Clinical Gastroenterology and Hepatology, 2006, vol. 4, no. 3, pp. 262-275.

18. Norton J.A., Ham C.M., Van Dam J., Jeffrey R.B., Longacre T.A., Huntsman D.G., Chun N., Kurian A.W., Ford J.M. CDH1 truncating mutations in the E-cadherin gene: an indication for total gastrectomy to treat hereditary diffuse gastric cancer. Annals of Surgery, 2007, vol. 245 no. 3, pp. 873-879.

19. Koea J.B., Karpeh M.S., Brennan M.F. Gastric cancer in young patients: demographic, clinicopathological, and prognostic factors in 92 patients. Annals of Surgical Oncology, 2000, vol. 7, no. 5, pp. 346-351.

20. Stiekema J., Cats A., Kuijpers A., van Coevorden F., Boot H., Jansen E.P., Verheij M., Balague Ponz O., Hauptmann M., van Sandick J.W. Surgical treatment 
results of intestinal and diffuse type gastric cancer. Implications for a differentiated therapeutic approach? European Journal of Surgical Oncology, 2013, vol. 39, no.7, pp. 686-693.

21. Francis W.P., Rodrigues D.M., Perez N.E., Lonardo F., Weaver D., Webber J.D. Prophylactic laparoscopic-assisted total gastrectomy for hereditary diffuse gastric cancer. Journal of the Society of Laparoendscopic Surgeons, 2007, vol. 11, no.1, pp. 142-147.

22. Choi J., Kim S.G., Im J.P., Kang S.J., Lee H.J., Yang H.K., Kim J.S., Kim W.H., Jung H.C., Song I.S. LymphnodemetastasisinmultiplesynLymph node metastasis in multiple synchronous early gastric cancer. Gastrointestinal Endoscopy, 2011, vol. 74, no.2, pp. 276-284.

23. Kang H.J., Kim D.H., Jeon T.Y., Lee S.H., Shin N., Chae S.H., Kim G.H., Song G.A., Kim D.H., Srivastava A., Parkdo Y., Lauwers G.Y. Lymph node metastasis from intestinal-type early gastric cancer: experience in a single institution and reassessment of the extended criteria for endoscopic submucosal dissection. Gastrointestinal Endoscopy, 2010, vol. 72, no. 3, pp. 508-515.

24. Carneiro F., Charlton A., Huntsman D. Hereditary diffuse gastric cancer. In: Bosman F.T., ed. WHO classification of tumours of the digestive system. $4^{\text {th }}$ edn. International Agency for Research on Cancer Scientific Publications, 2010, pp. 59-63.

25. Bosman F.T., Carneiro F., Hruban R.H., Theise N.D. WHO classification of tumours of the digestive system. $4^{\text {th }}$ edn. International Agency for Research on Cancer Scientific Publications, 2010.

26. Lauren P. The two histological main types of gastric carcinoma: diffuse and socalled intestinal-type carcinoma. An attempt at a histo-clinical classification. Acta pathologica, microbiologica, et immunologica Scandinavica, 1965, vol. 64, no.1, pp. 31-49.

27. Pandalai P.K., Lauwers G.Y., Chung D.C., Patel D., Yoon S.S. Prophylactic total gastrectomy for individuals with germline CDH1 mutation. Surgery, 2011, vol. 149 , no.3, pp. 347-355.

28. Bardram L., Hansen T.V., Gerdes A.M., Timshel S., Friis-Hansen L., Federspiel B. Prophylactic total gastrectomy in hereditary diffuse gastric cancer: identification of two novel $\mathrm{CDH} 1$ gene mutations-a clinical observational study. $\mathrm{Fa}$ milial Cancer, 2014, vol. 13, no. 2, pp. 231-242.

29. Carneiro F., Oliveira C., Suriano G., Seruca R. Molecular pathology of familial gastric cancer, with an emphasis on hereditary diffuse gastric cancer. Journal of Clinical Pathology, 2008, vol. 61, no.1: pp. 25-30.

30. Mills S.E. Histology for pathologists. 3rd edn. Philadelphia, USA: Lippincott Williams \& Wilkins, 2007, 1328 p.

31. Van der Kaaij M.A. et al, unpublished data, 2014. 
32. Van der Post et al, unpublished data, 2014.

33. Lee A.F., Rees H., Owen D.A., Huntsman D.G. Periodic acid-schiff is superior to hematoxylin and eosin for screening prophylactic gastrectomies from CDH1 mutation carriers. American Journal of Surgical Pathology, 2010, vol. 34, no. 7, pp. 1007-1013.

34. Oliveira C., Sousa S., Pinheiro H., Karam R., Bordeira-Carrico R., Senz J., Kaurah P., Carvalho J., Pereira R., Gusmao L., Wen X., Cipriano M.A., Yokota J., Carneiro F., Huntsman D., Seruca R. Quantification of epigenetic and genetic 2nd hits in CDH1 during hereditary diffuse gastric cancer syndrome progression. Gastroenterology, 2009, vol.136, no.7, pp. 2137-2148.

35. Majewski I.J., Kluijt I., Cats A., Scerri T.S., de Jong D., Kluin R.J., Hansford S., Hogervorst F.B., Bosma A.J., Hofland I., Winter M., Huntsman D., Jonkers J., Bahlo M., Bernards R. An alpha-E-catenin (CTNNA1) mutation in hereditary diffuse gastric cancer. Journal of Pathology, 2013, vol. 229, no. 4, pp. 621-629.

36. Worster E., Liu X., Richardson S., Hardwick R.H., Dwerryhouse S., Caldas C., Fitzgerald R.C. The impact of prophylactic total gastrectomy on health-related quality of life: a prospective cohort study. Annals of Surgery, 2014, vol.260, no. 1, pp. 87-93.

37. Mine S., Sano T., Tsutsumi K., Murakami Y., Ehara K., Saka M., Hara K., Fukagawa T., Udagawa H., Katai H. Large-scale investigation into dumping syndrome after gastrectomy for gastric cancer. Journal of the American College of Surgeons, 2010, vol. 211, no. 5, pp. 628-636.

38. Paik C.N., Choi M.G., Lim C.H., Park J.M., Chung W.C., Lee K.M., Jun K.H., Song K.Y., Jeon H.M., Chin H.M., Park C.H., Chung I.S. The role of small intestinal bacterial overgrowth in postgastrectomy patients. Neurogastroenterology \& Motility, 2011, vol. 23, no. 5, pp. e191-e196.

\section{ДАННЫЕ ОБ АВТОРАХ}

Быков Игорь Игоревич, врач-онколог онкологического отделения хирургических методов лечения опухолей головы и шеи, молочных желез, кожи, мягких тканей, кандидат медицинских наук ФГАОУ ВО Первый МГМУ им. И. М. Сеченова (Сеченовский Университет)

ул. Трубецкая, 8, стр. 2, г. Москва, 119991, Российская Федераџия igor-vr@mail.ru

Немцова Марина Вячеславовна, заведующая лабораторией медицинской генетики, профессор, доктор биологических наук 
ФГАОУ ВО Первый МГМУ им. И.М. Сеченова (Сеченовский Университет)

ул. Трубеикая, 8, стр. 2, г. Москва, 119991, Российская Федерация

Хоробрых Татьяна Витальевна, профессор по кафедре факультетской хирургии, врач-хирург онкологического хирургического отделения, доктор медицинских наук

ФГАОУ ВО Первый МГМУ им. И.М. Сеченова (Сеченовский Университет)

ул. Трубеикая, 8, стр. 2, г. Москва, 119991, Российская Федерачия

\section{DATA ABOUT THE AUTHORS}

Bykov Igor Igorevich, Oncologist, Oncology Department of Surgical Methods of Treatment of Tumors of Head and Neck, Mammary Glands, Skin, Soft Tissues, Cand. Sc. (Medicine)

I.M. Sechenov First Moscow State Medical University (Sechenov University)

8-2, Trubetskaya Str., Moscow, 119991, Russian Federation igor-vr@mail.ru

Nemtsova Marina Vyacheslavovna, Professor, Head of the Laboratory of Medical Genetics, D.Sc. (Biology)

I.M. Sechenov First Moscow State Medical University (Sechenov University)

8-2, Trubetskaya Str., Moscow, 119991, Russian Federation

Khorobrykh Tatiana Vitalevna, Professor, Department of Faculty Surgery, Oncologist, Oncology Surgical Department, D.Sc. (Medicine)

I.M. Sechenov First Moscow State Medical University (Sechenov University)

8-2, Trubetskaya Str., Moscow, 119991, Russian Federation 